whose role it is. Volunteers and support workers may be advantageous in having these conversations with the right support and development.

Methods There is a mixed methods approach to this research in two separate stages, involving 14 volunteers and support staff.

First stage Face to face qualitative interviews prior to exposure of new training/model of developing these conversations and pre-training Likert questionnaire have been undertaken.

Results Thematic analysis using NVIVO, to address study objectives from first stage, has identified variables in how participants engage with individuals prior to this support and training. Emerging themes are: discussions around whose role is it; feeling valued within their role; being given 'permission' and motivation around having these conversations.

Conclusions Second stage is now being undertaken, emerging themes will be presented in APM Conference. This stage will focus on identifying whether a different approach has helped, and volunteers' feedback will inform training developments and dissemination about how to support volunteers in having honest conversations about quality of life.

\section{FRAILTY SCORING IN PATIENTS WITH END STAGE RENAL FAILURE}

L Hetherington, J Prentice, M Findlay, T Collidge. NHS Greater Glasgow and Clyde, Scottish Renal Palliative Collaberative Group, The Glasgow Renal and Transplant Unit, South Glasgow University Hospital, The Beatson West of Scotland Cancer Centre, The University of Glasgow

\subsection{6/bmjspcare-2019-ASP.153}

Background In the end stage renal failure (ESRF) population frailty is associated with early mortality, increased hospitalisations, and significant symptom burden. After identifying a high degree of frailty in patients withdrawing from dialysis in our population we examined the use of formal frailty scoring and its use in identifying deteriorating patients on renal replacement therapy (RRT).

Methods The Rockwood Clinical Frailty Scale (CFS) is a 9point scale which enables measurement of frailty it has high inter-rater reliability and correlates well with objective measures of frailty and has been validated in the ESRF population. We introduced routine recording of the CFS for all ESRF in our region for patients at three monthly intervals and for low clearance patients at the time of RRT education.

Results A total of 1663 scores (range 1-9) have been recorded in 798 patients. Mean age 63.9 years. Of those patients currently undergoing haemodialysis the median CFS score was 4 $(n=533)$. The median score prior to death was 5.5. Evidence of deterioration in CFS score (last score greater than the first) was present in $50 \%(14 / 28)$ of those who were deceased at follow-up, whereas a deteriorating score was only present in $22.3 \%(97 / 435)$ of those who remained alive, $p=0.001$. A documented score of 6 or greater was present in $51.4 \%$ (38/ 74 ) of those who subsequently died vs. $21.7 \%(158 / 727)$ of those who remained alive, $\mathrm{p}<0.001$.

Conclusion Deterioration in frailty score is associated with death at follow-up. Furthermore, a score 6 or greater at any point is predictive of death at follow-up. Routine monitoring of frailty using the CFS provides a simple tool to identify patients who are deteriorating and at risk of death. High or deteriorating CFS score should trigger clinical review and anticipatory care planning where appropriate.

\section{A PROSPECTIVE COHORT STUDY DESCRIBING THE STABILITY OF CARE PREFERENCES IN FRAIL OLDER PEOPLE FOLLOWING ACUTE ILLNESS}

Simon Noah Etkind, Anna E Bone, Fliss EM Murtagh, Irene J Higginson. King's College London, Cicely Saunders Institute; Wolfson Palliative Care Research Institute, Hull York Medical School

\subsection{6/bmjspcare-2019-ASP.154}

Background Care should be responsive to preferences but there is little evidence regarding the stability of care preferences, particularly in frail older people after acute illness.

Aim To describe the stability of preferred care outcomes in frail older people in the six months following acute illness.

Methods Prospective cohort study of frail older adults with recent acute illness requiring secondary care review or admission, surveyed at three time-points over six months. Participants rated the importance of six preferred care outcomes chosen from literature review (to extend life, be comfortable, improve quality of life, remain independent, support others, stay out of hospital) on a 0-4 Likert scale, and were then asked to prioritise the most important. We used descriptive statistics to analyse preferences at baseline and the stability of preferences during the study.

Results 67 participants; 63\% female, mean age 84 (standard deviation 7.41). 10 participants (15\%) died during the study. At baseline, all preferred outcomes were rated mean $>3.3 / 4$ in importance, except 'to extend life' (2.08/ 4). Most frequent baseline priorities were: stay out of hospital (20\%), support others (16\%), and improve quality of life $(16 \%)$. During the study, the importance ascribed to each preferred outcome was largely stable, but what was most important changed at $66 \%$ of $n=85$ opportunities. Overall, being comfortable became most important for more patients during the study (increasing from 5\% to 14\%, (Chi2 4.41, p=0.036)), whilst there was a non-significant trend away from staying out of hospital being most important (27\% to $22 \%)$.

Conclusions For frail older people, the importance of preferred care outcomes remains stable after acute illness, but the outcome ranked as most important frequently changes. Due to its stability, the level of importance ascribed to preferred outcomes may be more useful in clinical practice and advance care planning.

\section{DESCRIBING DEPRESCRIBING - WHEN ARE WE STOPPING MEDICATIONS IN PALLIATIVE CARE?}

Matthew Doré, Hannah Fox, Trish Campbell, Derek Willis. Severn Hospice

10.1136/bmjspcare-2019-ASP.155

Introduction Deprescribing is the process of withdrawal of medication with the goal of improving patient care. Research currently is in the context of polypharmacy and geriatrics, however despite its ubiquity within palliative care there is a paucity of research in this context. Anecdotally, deprescribing is performed adhoc and using 'common sense' but there appears no clear data on rational, benefit and whole numbers involved.

Aim To gather data on current deprescribing practice within palliative care, with the aim to inform our own specialty and others. 